\title{
Banking DNA sequences
}

MOUNT Everest, as we all know, was first climbed "because it was there". The Everest of those scientists who are devoted to the sequencing of DNA is the complete human genome, a peak that cannot yet be conquered. Nevertheless it seems not out of the question, even now, to attempt to sequence a single chromosome from man or Drosophila; or at least to tackle a substantial fragment of one such chromosome. And certainly some of the more ambitious sequencers would no longer consider the complete genome of the bacterium Escherichia coli to be a sufficient challenge to their skills.

The reasons why grandiose sequencing can now be contemplated are technical. In the early 1960 s when Walter Fiers embarked upon the, then, highly ambitious project of sequencing the first complete genome, that of the bacterial virus MS2, it was not possible to sequence fragments of longer than eight nucleotides at one time. The complete structure of the 3,569 nucleotide genome of MS2 was published in 1976. Now, thanks largely to techniques developed by Alan Maxam and Walter Gilbert in Harvard and by Fred Sanger and his colleagues in Cambridge, one can sequence stretches of up to 250 nucleotides at a time. The sequencing of MS2 would now be no more than a single $\mathrm{PhD}$ project in a laboratory where the necessary techniques were already in operation.

Rapid sequencing, however, is only half the trick. One needs also relatively large amounts of pure DNA to be sequenced. For that, the techniques of cloning recombinant DNA in bacteria have been invaluable.

The combination of rapid sequencing and cloning has made it feasible to tackle larger and larger genomes. Following MS2, there came the bacterial virus $\Phi \times 174$ and the mammalian SV40, polyoma and hepatitis viruses. In the offing is the complete sequence of several more viruses, led by adenovirus, and that of the genome of mammalian and yeast mitochondria. In quantitative terms this is progress from the 3.6 kilobases (one thousand nucleotides) of MS2 to the 5.4 kilobases of $\Phi \times 174$, through the 35 kilobases of adenovirus to the 50 kilobases of yeast mitochondria.

By contrast the complete genome of Escherichia coli is about 10,000 kilobases, the Drosophila genome an order of magnitude greater and a single human chromosome some 500,000 kilobases of DNA. With a realistic outlook the Escherichia coli sequence could be completed by a team of ten in less than five years; with an optimistic one, a tenth of a human chromosome would not take as long because the very thought of devoting a lifetime to the task would force an order of magnitude increase in the speed of sequencing!

The dilemma for sequencers deciding what to tackle next is that, whereas a new graduate in the right laboratory might immediately stumble upon an iconoclastic stretch of DNA, so might a bevy of experts take several years to reveal nothing more than another 1,000 kilobases of uninteresting sequence.

Not that it is possible to overestimate the astonishing discoveries that have emerged, unsuspected, from sequencing: overlapping genes, split genes, degenerate genes and the bizarre features of the mitochondrial genome, including a genetic code that breaks the rule that the code is universal.

As with so much in science, hunches may be the only way to make decisions, be they by project leaders or grant givers.

From any large scale sequencing there now arises a series of irritating problems. The first is in what quanta to report the sequence; gene by gene or only when the sequence of the whole genome is essentially complete? In theory the latter approach is preferable, unless any particularly interesting feature is revealed along the way. But can the pressure to milk a sequence for as many papers as possible, or even just one for each collaborator, be resisted?

Then there is the problem, faced by journals, of printing screeds of ATCG permutations, less appetising to those who have not acquired the taste, than even the stock exchange page of a newspaper. The temptation - perhaps to be resisted - is to photo-reduce them to near-illegibility. Worse only is the suggestion, sometimes voiced, that sequences should be available from the author, but not be published at all. That would serve the thinking reader about as well as does television's The Forsyte Saga act as a guide to Galsworthy's literary style.

Finally, one major problem arises from the quantity of sequencing that has already been amassed - of the order of 100 kilobases - let alone that which the next few years will bring. The problem is how best to collate as much data as possible so as to bring out salient features. Although number, or rather letter, crunching is no substitute for thought, nevertheless computers are an essential aid to sequence collation. Already they are needed in any large scale sequencing simply to piece together the data obtained from the sequencing of random fragments of large genetic units. What does not yet exist is any central data bank in which all sequences could be deposited and made freely available to the whole scientific community. There are good precedents for such a service in the Brookhaven Protein Data Bank and the Cambridge Crystallographic Data Centre. The need for a DNA sequence bank is becoming urgent. At its best it would operate on a computer network system and offer search programme facilities. At its most meagre it would be no more than a depository, run by a keyboard operator, which sent out tapes for a nominal fee. Proposals to start some kind of bank in the US have been in the air for at least a year, and last week the European Molecular Biology Laboratory veered towards a decision to start one. It matters little by whom, or where, a bank is started. What is important is that it should start soon. 